\title{
Dynamics of Race Structures of the Rice Blast Pathogen Population in Heilongjiang Province, China From 2006 Through 2015
}

\author{
Yaling Zhang, ${ }^{1,2}$ Jinyan Wang, ${ }^{1}$ Yongxiang Yao, ${ }^{1,3}$ Xuehui Jin, ${ }^{2}$ James Correll, ${ }^{4}$ Ling Wang, ${ }^{1}$ and Qinghua Pan ${ }^{1, \dagger}$ \\ ${ }^{1}$ State Key Laboratory for Conservation and Utilization of Subtropic Agrobioresurces, Guangdong Provincial Key Laboratory for \\ Crop Molecular Breeding, College of Agriculture, South China Agricultural University, Guangzhou 510642, China \\ ${ }^{2}$ College of Agronomy, Heilongjiang Bayi Agricultural University, Daqing 163319, China \\ ${ }^{3}$ Dandong Academy of Agricultural Sciences, Dandong 118109, China \\ ${ }^{4}$ Department of Plant Pathology, University of Arkansas, Fayetteville 72701, AR, U.S.A.
}

\begin{abstract}
Rice blast caused by the fungus Magnaporthe oryzae is one of the most destructive diseases of rice. Its control through the deployment of host resistance genes would be facilitated by understanding the pathogen's race structure. Here, dynamics of race structures in this decade in Heilongjiang province were characterized by Chinese differential cultivars. Two patterns of dynamics of the race structures emerged: both race diversity and population-specific races increased gradually between 2006 and 2011, but they increased much more sharply between 2011 and 2015,

the race structure remained stable through this decade. On the host side, the composition of resistance in the cultivar differential set could be divided in two: the three indica-type entries of the differential set expressed a higher level of resistance to the population of M. oryzae isolates tested than did the four japonica-type entries. The cultivars Tetep and Zhenlong 13 as well as two additional resistance genes $\alpha$ and $\varepsilon$ were confirmed as the most promising donors of blast resistance for the local rice improvement programs.
\end{abstract} with concomitant falls in both the population-common races and dominant races. Four races (ZD1, ZD3, ZD5, and ZE1) were among the top three dominant races over the whole period, indicating that the core of
Keywords: cereals and grains, disease development and spread, disease management, epidemiology, field crops
Pathogen races (also referred to as physiological races or pathotypes) are defined by their interaction with a set of host cultivars that each carry a distinct profile of known major resistance genes (Atkins et al. 1967; Feng et al. 2018; Kolmer and Hughes 2018; Liu et al. 2017; Wang et al. 2017). Although a number of studies have addressed the race structure of certain plant pathogens, little attention has been paid to simultaneously characterizing both temporal and spatial dynamics of race structures (Chen et al. 2001; Dorrance et al. 2016; Kawasaki-Tanaka et al. 2016; Kolmer and Hughes 2018; Liu et al. 2017; Wang et al. 2017; Yang et al. 2004; Zhang et al. 2017). Host resistance is often conditioned by a single gene. It tends to follow the gene-for-gene model so that, when the resistance gene no longer recognizes a pathogen's avirulence gene owing to a change in that gene, the result is an abrupt loss in resistance, and disease ensues (Flor 1971; Wu et al. 2014; Zhang et al. 2015). Concomitantly, the deployment of new host resistance genes drives

\author{
${ }^{\dagger}$ Corresponding author: Q. Pan; panqh@scau.edu.cn \\ Y. Zhang, J. Wang, and Y. Yao contributed equally to this work.
}

Funding: We acknowledge funding from National Key R\&D Project grant 2016YDF0100601; National Transgenic Research Project grant 2016ZX08001-002; Guangzhou Key R\&D Project grant 201504010029; National Natural Science Foundation of China grants 31870137, 31471175, and U1131003; Guangdong Province Key Laboratory of Plant Molecular Breeding grant GPKLPMB201801; and Natural Science Foundation of Heilongjiang Province grant QC2011C046.

*The $\boldsymbol{e}$-Xtra logo stands for "electronic extra" and indicates that three supplementary tables are published online.

The author(s) declare no conflict of interest.

Accepted for publication 6 June 2019

Copyright $\odot 2019$ The Author(s). This is an open access article distributed under the CC BY-NC-ND 4.0 International license. alterations in a pathogen's race structure (Mekwatanakarn et al. 2000; Wang et al. 2017; Wu et al. 2014; Zhang et al. 2015, 2017). Characterizing and monitoring the dynamics of pathogen population race structures can contribute to devising a sustainable strategy to control plant disease through the deployment of appropriate resistance genes (Hu et al. 2002; Kawasaki-Tanaka et al. 2016; Kolmer and Hughes 2018; Liu et al. 2017; Wang et al. 2017; Zhang et al. 2017).

The largest rice production area in China is in the northeastern province of Heilongjiang (HLJ). The area planted locally to rice increased from 1.99 million hectares (Mha; $6.9 \%$ of the national rice production area) in 2006 to 4.01 Mha $(13.3 \%)$ in 2015 ; in terms of production, grain harvest has risen from 13.6 million tons $(\mathrm{Mt}$; $7.5 \%$ of the national total) to $22.0 \mathrm{Mt}$ (10.6\%) (Zhang 2018). Rice blast caused by the heterothallic ascomycete Magnaporthe oryzae represents one of the most serious biotic constraints on yield in rice (Wang et al. 2017; Zhang et al. 2017; Zhao et al. 2018). Although a previous study compared the race structure of the HLJ M. oryzae population with that present in other parts of China (Zhang et al. 2017), the focus of this investigation was the characterization of the dynamic behavior of the HLJ M. oryzae population over the previous decade. To this end, the interaction between $M$. oryzae isolates sampled at various time points during the period 2006 through 2015 and a set of Chinese differential cultivars (CDCs) was tested. At the same time, the $M$. oryzae resistance genes present in the differential set of cultivars was defined, thereby providing direct information to rice breeders regarding the resistance genes being used in the rice breeding programs during the period.

\section{Materials and Methods}

Pathogen isolate and inoculum preparation. Diseased rice panicles were sampled from fields across HLJ from 2006 to 2015. Standard methods (Zhang 2018; Zhang et al. 2017) were used to both derive and store single-spore isolates of $M$. oryzae from these samples. The race structures of the three populations Pop-06, Pop-11, and Pop- 15 were subjected to investigation of race structure dynamics over a decade. Pop- 06 consisted of 97 isolates that were recovered from 269 panicle samples collected from 10 counties; Pop-11 
contained 103 isolates recovered from 219 samples collected from 11 counties, and Pop-15 contained 96 isolates recovered from 317 samples collected in 19 counties (Supplementary Table S1) (Zhang 2018).

Inoculation with M. oryzae and scoring for disease. Inoculum of each isolate was prepared as described in Pan et al. (1996) and modified by Zhang (2018) and Zhang et al. (2017). The concentration of the inoculum suspension was adjusted to 10 to $50 \times 10^{4}$ conidia per milliliter for inoculation. The CDC set, which comprised three indica-type entries (cultivars Tetep, Zhenong 13, and Sifeng 43) along with four japonica-type entries (cultivars Dongnong 363, Kando 51, Hejiang 18, and Lijiangxintuanheigu [LTH]), was challenged with each of the 296 M. oryzae isolates. Each CDC entry was represented by 10 hydroponically raised seedlings following methods described by Zhang (2018). The inoculum was sprayed on seedlings at the four- to five-leaf stage. The host reaction was scored 6 days postinoculation using the Pan et al. (1996) numerical scale (0 to 2: resistant; 3 to 5: susceptible). Inoculation of each CDC entry with each isolate was carried out at least twice in independent inoculations. In cases of inconsistency, the highest score recorded for the host reaction was used.

Race identification. Each of the seven CDC entries was assigned a race code (All China Corporation of Research on Physiological Races of Pyricularia oryzae 1980), which combines both an alphabetical component and a numerical component, namely Tetep, race code A64; Zhenlong 13, race code B32; Sifeng 43, C16; Dongnong 363, D8; Kando 51, E4; Hejiang 18, F2; and LTH, G1 (Supplementary Table S2). A given isolate of $M$. oryzae was initially assigned its race group according to its compatible reaction produced on the alphabetically upper CDC entry (from A to G), after which race number (a numerical value) was assigned, representing the sum of the codes corresponding to the remaining entries on which an incompatible reaction was observed, followed by adding 1 to the numerical value to avoid coding races as $\mathrm{A} 0$ and $\mathrm{B} 0$. The suffix $\mathrm{H}$ was assigned to all isolates that were incompatible with all seven CDC entries (All China Corporation of Research on Physiological Races of Pyricularia oryzae 1980; Zhang et al. 2017).

Data analysis. Race structure was dissected into four aspects with nine parameters (Supplementary Table S3). The total race frequency $f_{t r}[(t r / N) \times 100 \%]$ and the race diversity index $h_{r d i}[(N / N-1)(1-$ $\left.\Sigma x_{i}^{2}\right)$ ] were used to represent race diversity; in the latter case, the index gets closer to one as the population becomes more diverse: the population common race frequency $f_{p c r}[(p c r / t r) \times 100 \%]$, the population common race isolate frequency $f_{p c r i}[(p c r i / N) \times 100 \%]$, and the total population common race isolate frequency $f_{t p c r i}[(t p c r i / N) \times$ $100 \%$ ] for common race structure; the population specific race frequency $f_{p s r}[(p s r / t r) \times 100 \%]$ and the population specific race isolate frequency $f_{p s r i}[(p s r i / N) \times 100 \%]$ for specific race structure; and the top three race isolate frequency $f_{t 3 r i}[(t 3 r i / N) \times 100 \%]$ and the total top three race isolate frequency $f_{t t 3 r i}[(t t 3 r i / N) \times 100 \%]$ for dominant race structure. In these expressions, $N$ represented the number of the isolates within a population, $t r$ represented the number of races identified within a given population, $x_{i}$ represented the frequency of race

Table 1. Race diversity of three populations of Magnaporthe oryzae from Heilongjiang province recovered from symptomatic rice panicles over the period from 2006 to 2015 assessed by challenging a set of Chinese differential cultivars

\begin{tabular}{|c|c|c|c|c|}
\hline Parameter & $2006(97)^{a}$ & $2011(103)$ & & $2015(96)$ \\
\hline No. of race group & 6 & 6 & & 7 \\
\hline No. of race & 12 & 14 & & 25 \\
\hline Race frequency $\left(f_{t r}\right)$ & 12.4 & 13.6 & & 26.0 \\
\hline Race diversity index $\left(h_{r d i}\right)$ & 0.800 & 0.813 & & 0.938 \\
\hline$\chi^{2}$ for $f_{t r} \mathrm{~b}$ & & 0.06 & $3.88^{\mathrm{c}}$ & \\
\hline
\end{tabular}

${ }^{a}$ Numbers shown in parentheses represent the numbers of isolates tested.

b The $\chi^{2}$ test was applied to pairwise comparison for Pop-11 versus Pop-06 and Pop-11 versus Pop-15 for the first and second half of the decade, respectively.

c Significant at the $P=0.05$ level for a $\chi^{2}$.
$\# i$, pcr represented the number of population-common races across all three populations, pcri represented the number of populationcommon race isolates within a population, tpcri represented the total number of population-common race isolates within a population, $p s r$ represented the number of population-specific races within a population, $p s r i$ represented the number of population-specific race isolates within a population, $t 3 r i$ represented the number of top three race isolates within a population, and $t t 3 r i$ represented the total number of the top three race isolates within a population. To test a hypothesis that the race structure has not changed in this decade in HLJ, a $\chi^{2}$ test was applied to pairwise comparison for Pop-11 versus Pop-06 and Pop11 versus Pop-15 for the first and second half of the period, respectively, for the key parameters.

The dynamic behavior of the host resistance gene content was quantified using the parameters cultivar resistance frequency $f_{C R}$ $[(C R I / N) \times 100 \%]$ and the total cultivar resistance frequency $f_{T C R}$ [(TCRI/TN $) \times 100 \%]$, where $C R I$ represented the number of isolates that were avirulent on a given CDC entry, TCRI represented the total number of isolates that were avirulent across all three populations, and $T N$ represented the total number of isolates of all three populations (Zhang et al. 2017). For clarity, each CDC entry was given an overall resistance rating (high: $>85 \%$; intermediate: 60 to $84 \%$; and low: $<60 \%$ ) following Zhang et al. (2017).

\section{Results}

Race diversity. Of the three $M$. oryzae populations, Pop-15 was more diverse than either Pop-06 or Pop-11. The 25 races detected in Pop-15 were distributed across seven of the eight groups, featuring an $f_{t r}$ value of $26.0 \%$ (Table 1). Within both Pop-06 (12 races detected) and Pop-11 (14 races), six of the eight groups were represented, equivalent to $f_{t r}$ values of 12.4 and $13.6 \%$, respectively. The $h_{r d i}$ values were consistent with those of $f_{t r}$, namely 0.800 (Pop-06), 0.813 (Pop-11), and 0.947 (Pop-15). Thus, there was an increase in race frequency and diversity during the first half of the decade and an even greater increase in race frequency and diversity during the second half of the decade.

Common race structure. In all, nine races (ZB25, ZB33, ZB57, $\mathrm{ZC} 1, \mathrm{ZC}$, ZD1, ZD5, ZE1, and ZF1) were identified as being represented in all three populations, leading to estimated $f_{p c r}$ values of $75.0 \%$ in Pop-06, 64.3\% in Pop-11, and 36.0\% in Pop-15 (Tables 1 and 2). The $f_{p c r i}$ values for the set of shared races varied from 32.0 (ZD1 in Pop-06) to $1.0 \%$ (ZF1 in Pop-06; ZC1 in Pop-11; and ZB25, ZB33, and ZB57 in Pop-15) (Table 2). The estimated $f_{t p c r i}$ values were $93.8 \%$ in Pop-06, 90.3\% in Pop-11, and 51.0\% in Pop15. The proportions of the common races were greater from Pop-06 to Pop-11 and considerably lower from Pop-11 to Pop-15, indicating

Table 2. Common races of Magnaporthe oryzae in three populations of pathogen recovered from the Heilongjiang region over the period from 2006 to 2015

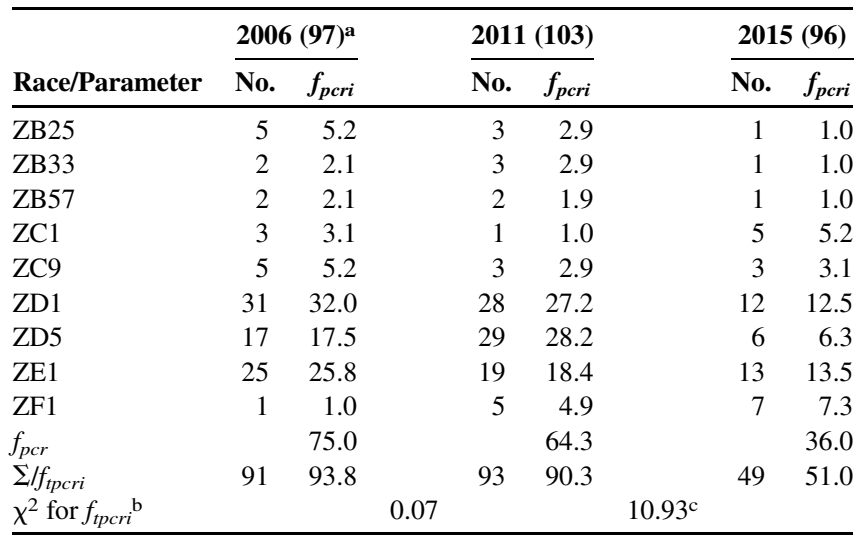

${ }^{\text {a }}$ Figures shown in parentheses represent the number of isolates tested.

b The $\chi^{2}$ test was applied to pairwise comparison for Pop-11 versus Pop-06 and Pop-11 versus Pop-15 for the first and second half of the decade, respectively.

${ }^{c}$ Significant at the $P=0.001$ level for a $\chi^{2}$. 
that race structures in both Pop-06 and Pop-11 were stable and changed in Pop-15.

Specific race structure. The numbers of population-specific races in Pop-06, Pop-11, and Pop-15 were one, four, and 13, respectively, resulting in estimated $f_{p s r}$ values of $8.3,28.6$, and $52.0 \%$, respectively (Tables 1 and 3). The numbers of isolates were one (Pop-06), seven (Pop-11), and 42 (Pop-15), giving rise to $f_{p s r i}$ values of $1.0,6.8$, and $42.7 \%$, respectively. Thus, the population-specific races increased steadily from Pop06 to Pop-15, but population-specific race isolates increased gradually from Pop-06 to Pop-11 and more abruptly from Pop-11 to Pop-15.

Dominant race structure. Three group D races (ZD1, ZD3, and ZD5) and one group E race (ZE1) emerged as the dominant races across the three populations (Table 4). The $f_{t 3 r i}$ values ranged from 32.0 to $9.4 \%$, whereas the $f_{t t 3 r i}$ values were $75.5 \%$ (Pop-06), $73.8 \%$ (Pop-11), and 35.4\% (Pop-15). ZD1 and ZE1 were the dominant races in all three populations: ZD1 represented $32.0 \%$ of Pop-06, $27.2 \%$ of Pop-11, and $12.5 \%$ of Pop-15; ZE1 was dynamic among the three populations (Table 4). Considering the entire decade, the dominant races were limited to very few races; however, their proportions were slightly reduced from Pop-06 to Pop-11 and greatly reduced from both to Pop-15.

Resistance structure. The resistance gene composition of the $\mathrm{CDC}$ entries was based on the conclusions drawn by Zhang et al.
(2017). The level of resistance displayed by the subsets of indica and japonica types differed markedly when challenged by each of the three $M$. oryzae populations (Table 5). Among the indica types, Tetep and Zhenlong 13 expressed a greater level of resistance than other entries, irrespective of which M. oryzae population was involved, whereas Sifeng 43 proved to be more resistant when challenged by isolates from Pop-06 than when challenged by isolates from either Pop-11 or Pop-15. All of the japonica-type entries performed poorly when challenged with isolates from each of the $M$. oryzae populations; LTH was susceptible to every isolate. Zhenlong 13 was more resistant to M. oryzae than Dongnong 363, possibly reflecting the presence of the resistance gene $\varepsilon$. The difference between the reactions of Sifeng 43 and Hejiang 18 (especially when challenged by the isolates in Pop-06 and Pop-11) implied that the combination $P i b+\alpha$ imparted a superior level of resistance than was provided by Pia on its own. Collectively, the resistance composition of the CDC set in this decade reacted differently in terms of resistance between the indica and japonica subsets to inoculation with the isolates of $M$. oryzae.

\section{Discussion}

The race structure of the population of $M$. oryzae in the largest rice cropping area, HLJ, has been repeatedly investigated (Gao et al.

Table 3. Specific races of Magnaporthe oryzae in three populations of pathogen recovered from the Heilongjiang region over the period from 2006 to 2015

\begin{tabular}{|c|c|c|c|c|c|c|}
\hline \multirow[b]{2}{*}{ Population } & \multirow[b]{2}{*}{ Specific race (no. of isolates) ${ }^{a}$} & \multicolumn{2}{|c|}{ Race } & \multicolumn{2}{|c|}{ Isolate } & \multirow[b]{2}{*}{$\chi^{2}$ for $f_{p s r i}{ }^{\mathrm{b}}$} \\
\hline & & No. & $f_{p s r}$ & No. & $f_{p s r}$ & \\
\hline $2006(97)$ & ZB49 (1) & 1 & 8.3 & 1 & 1.0 & \\
\hline & & & & & & $4.31^{\mathrm{c}}$ \\
\hline 2011 (103) & ZA9 (1), ZB37 (2), ZB41 (2), ZC5 (2) & 4 & 28.6 & 7 & 6.8 & $27.06^{\mathrm{d}}$ \\
\hline $2015(96)$ & $\begin{array}{l}\text { ZA21 (1), ZB1 (2), ZB3 (1), ZB5 (3), ZB7 (1), ZB19 (1), ZC3 (3), } \\
\text { ZC7 (2), ZC11 (2), ZD3 (9), ZD7 (8), ZE3 (4), ZG1 (5) }\end{array}$ & 13 & 52.0 & 42 & 43.8 & \\
\hline
\end{tabular}

${ }^{a}$ Races are ordered according to their alphanumeric code.

b The $\chi^{2}$ test was applied to pairwise comparison for Pop-11 versus Pop-06 and Pop-11 versus Pop-15 for the first and second half of the decade, respectively.

c Significant at the $P=0.05$ level for a $\chi^{2}$.

d Significant at the $P=0.001$ level for a $\chi^{2}$.

Table 4. Dominant race of Magnaporthe oryzae in three populations of pathogen recovered from the Heilongjiang region over the period from 2006 to 2015

\begin{tabular}{|c|c|c|c|c|c|c|c|c|}
\hline \multirow[b]{2}{*}{ Population } & \multicolumn{6}{|c|}{ Top 3 races and their frequencies } & \multirow[b]{2}{*}{ Top $3 f_{t t 3 r i}$} & \multirow[b]{2}{*}{$\chi^{2}$ for $f_{t t 3 r i}{ }^{a}$} \\
\hline & First (isolates) & $f_{t 3 r i-1}$ & Second (isolates) & $f_{t 3 r i-2}$ & Third (isolates) & $f_{t 3 r i-3}$ & & \\
\hline $2006(97)$ & ZD1 (31) & 32.0 & ZE1 (25) & 25.8 & ZD5 (17) & 17.5 & 75.3 & \\
\hline $2011(103)$ & ZD5 (29) & 28.2 & ZD1 (28) & 27.2 & ZE1 (19) & 18.4 & 73.8 & 0.02 \\
\hline $2015(96)$ & ZE1 (13) & 13.5 & ZD1 (12) & 12.5 & ZD3 (9) & 9.4 & 35.4 & \\
\hline
\end{tabular}

a The $\chi^{2}$ test was applied to pairwise comparison for Pop-11 versus Pop-06 and Pop-11 versus Pop- 15 for the first and second half of the decade, respectively.

b Significant at the $P=0.01$ level for a $\chi^{2}$.

Table 5. Resistance genes and resistance frequencies of a set of Chinese differential cultivar entries that were challenged with isolates of Magnaporthe oryzae recovered from the Heilongjiang region over the period from 2006 to 2015

\begin{tabular}{|c|c|c|c|c|c|c|}
\hline \multirow[b]{2}{*}{ Differential cultivar } & \multirow[b]{2}{*}{ Race code } & \multirow[b]{2}{*}{ Resistance gene } & \multicolumn{3}{|c|}{ Resistance frequency $\left(f_{C R}\right)$} & \multirow{2}{*}{$\begin{array}{c}\text { Total resistance } \\
\text { frequencies }\left(f_{T C R}\right)\end{array}$} \\
\hline & & & 2006 & 2011 & 2015 & \\
\hline Tetep & A64 & Pil, Pi4, Pi54 & $93.8^{\mathrm{a}}$ & $90.3^{\mathrm{a}}$ & $95.8^{\mathrm{a}}$ & $93.2^{\mathrm{a}}$ \\
\hline Zhenlong 13 & B32 & Pik, Pia, $\beta, \varepsilon$ & $89.7^{\mathrm{a}}$ & $96.1^{\mathrm{a}}$ & $86.5^{\mathrm{a}}$ & $90.9^{\mathrm{a}}$ \\
\hline Sifeng 43 & $\mathrm{C} 16$ & Pib, Pia, $\alpha$ & $89.7^{\mathrm{a}}$ & 83.5 & 74.0 & 82.4 \\
\hline Dongnong 363 & D8 & Pik, Pia, $\beta$ & 39.2 & 36.9 & 39.6 & 38.5 \\
\hline Kando 51 & E4 & $P i k, \gamma$ & 18.6 & 39.8 & 36.5 & 31.8 \\
\hline Hejiang 18 & $\mathrm{~F} 2$ & Pii, Pia, $\delta$ & 0.0 & 0.0 & 37.5 & 12.2 \\
\hline Lijiangxintuanheigu & G1 & Pik-l & 0.0 & 0.0 & 0.0 & 0.0 \\
\hline
\end{tabular}

${ }^{a}$ Higher levels of resistance. 
2017; Lei et al. 2011; Li and Zheng 1989; Xin et al. 2016; Zhang et al. 2009 ), but the influence of season and location on the dynamic behavior of the race structure has not been well defined. In our previous research, the spatial features of the race structure of $M$. oryzae in HLJ were refined on the basis of comparison with other Chinese $M$. oryzae populations (Zhang et al. 2017). In this study, the race structures of three populations of $M$. oryzae recovered from HLJ were temporally characterized by the four aspects with the nine comparable parameters: that is, $f_{t r}$ and $h_{r d i}$ for rice diversity (Table 1); $f_{p c r}, f_{p c r i}$, and $f_{t p c r i}$ for common race structure (Table 2); $f_{p s r}$ and $f_{p s r i}$ for specific race structure (Table 3 ); and $f_{t 3 r i}$ and $f_{t t 3 r i}$ for dominant race structure (Table 4). This allowed for the recognition of two distinct phases in the development of the population race structure of M. oryzae in HLJ: both race diversity and the population-specific races increased gradually between 2006 and 2011, but the increase was much greater between 2011 and 2015, with concomitant decreases in both the number of population-common races and dominant races. Similarly, Liu et al. (2017) provided evidence of an acceleration in the appearance of new races of the wheat stripe rust pathogen in the United States. They detected 171 races of the pathogen between 1968 and 2009 , but from 2000 onward, the number of races detected increased at a greater rate than in the previous decades.

The biological basis for the change in population race structure that we detected may reside in the number of rice cultivars cultivated in HLJ over the course of the study period. Whereas 53 cultivars were grown over an extensive area at the beginning of the period of investigation (2006), this number had increased to 92 by 2011, and in 2015, 127 cultivars were planted (Zhang 2018). The time lag observed between the growth of race numbers and the increase in the number of host cultivar probably reflects the time needed for the pathogen to adapt to a more complex spectrum of host resistance genes. Similarly, an analysis of $\sim 1,000 \mathrm{M}$. oryzae isolates collected in United States over the period from 1959 to 2015 suggested that race structure varied more over time than over space, leading to the conclusion that host-driven selection is the major determinant of this pathogen's variability (Wang et al. 2017).

Interestingly, in our study, just four races (ZD1, ZD3, ZD5, and ZE1) (Table 4) dominated the population of M. oryzae in HLJ throughout the period of study, indicating a degree of stability in the pathogen's race structure, although the $f_{t t 3 r i}$ changed considerably from the first to second half of the period. These data infer that the core resistance genetics of the host remained stable and included the resistance genes Pia, Pii, and Pik that were all adopted from Japanese cultivars in 1970s in HLJ. That is, these cultivars have served as key parents in the local rice breeding programs for over five decades (Sun 2011; Zhang 2018; Zhang et al. 2017). Similar observations of the limited dominate races in the pathogen populations were reported in other groups (Liu et al. 2017; Mekwatanakarn et al. 2000; Wang et al. 2017).

Differential cultivar sets are founded on the principle of the genefor-gene hypothesis (Atkins et al. 1967; Flor 1971; KawasakiTanaka et al. 2016; Liu et al. 2017; Zhang et al. 2017). These cultivar sets provide data regarding the race or pathotype structure of the pathogen and also, what resistance genes/genotypes are being used or could be effective in local host cultivars (Liu et al. 2017; Zhang et al. 2017). Lei et al. (2011) recommended that cultivars grown in HLJ contain the genes Pi9, Piz-5 (Pi2), Pita-2, Pi12, and $P i 20$ based on the disease response of a set of 31 entries, each carrying a single major $M$. oryzae resistance gene, and a group of 12 popular local cultivars. Similarly, Kawasaki-Tanaka et al. (2016) reported that 16 resistance genes varied in their effectiveness against Japanese populations of $M$. oryzae. They reported that the presence of Pit ensured resistance against the full set of isolates tested; note that this gene was not fully effective against populations of M. oryzae found in China (Zhang et al. 2017). In the soybean root rot pathosystem, Dorrance et al. (2016) reported that none of the resistance genes involved in the 12 soybean differentials were effective against all of the isolates tested, but Rps 6 and $R p s 8$ were effective against the majority of isolates collected in northern regions of the sampled area in the United States. In the stripe rust pathosystem, Liu et al. (2017) identified two resistance genes $Y r 5$ and Yr15 from 18 monogenic differential lines, and both have conveyed stable resistance against the cognate pathogen since the 1960 s in the United States.

In this research, we demonstrated that the dynamics of resistance within the CDCs could be divided into two types: the three indica entries expressed a higher resistance than the four japonica entries did (Table 5). The two entries Tetep and Zhenlong 13 seemed to be particularly promising donors of $M$. oryzae resistance for HLJ rice improvement programs, because they were resistant to most of the isolates tested. Based on the differential reactions of Sifeng 43 and Hejiang 18 as well as those of Zhenlong 13 and Dongnong 363, the presence of the two putative resistance genes $\alpha$ and $\varepsilon$ was also confirmed (Table 5). Similarly, in our previous study, a total of five additional resistance genes, $\alpha$ to $\varepsilon$, were identified from the CDC based on spatial comparison of resistance performance among 7 CDCs and 12 Japanese differential cultivars with four populations of $M$. oryzae from China (Zhang et al. 2017) (Table 5). The three major resistance genes (Pia, Pii, and Pik) carried by the japonica subset of the CDCs might serve as a core of resistance in cultivars grown in HLJ over the whole period, because the four dominant races detected in 2006 to 2015 were responsible for reactions on the japonica subset carrying the three genes (Table 5). It would also be worthwhile to more thoroughly investigate the core of resistance within a given region and incorporate promising resistance genes into future cultivars to ensure a sustainable level of control over the disease (Dorrance et al. 2016; Liang et al. 2016; Lin et al. 2007; Mundt 2018).

\section{Literature Cited}

All China Corporation of Research on Physiological Races of Pyricularia oryzae. 1980. Research on physiological races of rice blast fungus in China. Acta Phytopathol. Sin. 10:71-82.

Atkins, J., Robert, A., Adair, C., Goto, K., Kozaka, T., Yanagida, R., Yamada, M., and Matsumoto, S. 1967. An international set of rice varieties for differentiating races of Piricularia pryzae. Phytopathology 57:297-301.

Chen, H., Chen, B., Zhang, D., Xie, Y., and Zhang, Q. 2001. Pathotypes of Pyricularia grisea in rice fields of central and southern China. Plant Dis. 85: 843-850.

Dorrance, A., Kurle, J., Robertson, A., Bradley, C., Giesler, L., Wise, K., and Concibido, V. 2016. Pathotype diversity of Phytophthora sojae in eleven states in the United States. Plant Dis. 100:1429-1437.

Feng, C., Saito, K., Liu, B., Manley, A., Kammeijier, K., Mauzey, S., Koike, S., and Correll, J. 2018. New races and novel strains of the spinach downy mildew pathogen Peronospora effuse. Plant Dis. 102:613-618.

Flor, H. 1971. Current status of the gene for gene concept. Annu. Rev. Phytopathol. 9:275-296.

Gao, X., Jin, Z., Li, Z., Zhang, X., Han, B., and Zhang, Z. 2017. Identification of rice blast pathogen races in Heilongjiang in 2015. China Rice 23:50-53.

Hu, Z., Chen, J., Wang, L., Qiu, Q., and Pan, Q. 2002. Molecular genetic studies on the rice blast fungus population. II. Temporal and spatial characterization of genetic structure of the fungus population in 2000 in Guangdong province. Mycosystema 21:203-209.

Kawasaki-Tanaka, A., Hayashi, N., Yanagihara, S., and Fukuta, Y. 2016. Diversity and distribution of rice blast (Pyricularia oryzae Cavara) races in Japan. Plant Dis. 100:816-823.

Kolmer, J., and Hughes, M. 2018. Physiologic specialization of Puccinia triticina on wheat in the United States in 2016. Plant Dis. 102:1066-1071.

Lei, C., Zhang, G., Cheng, Z., Ma, J., Wang, J., Xin, A., Chen, P., Xiao, J., Zhang, X., Liu, Y., Guo, X., Wang, J., Zhai, H., and Wan, J. 2011. Pathogenic races and virulence gene structure of Magnaporthe oryzae population and rice breeding strategy for blast resistance in Heilongjiang province. Acta Agron. Sin. 37: $18-27$.

Li, H., and Zheng, G. 1989. Studies on dynamics of races of Magnaporth oryzae populations collected in Heilongjiang province, China. Plant Prot. 51:2-4

Liang, Z., Wang, L., and Pan, Q. 2016. A new recessive gene conferring resistance against rice blast. Rice 9:47.

Lin, F., Liu, Y., Wang, L., Liu, X., and Pan, Q. 2007. A high-resolution map of the rice blast resistance gene Pi15 constructed by sequence-ready markers. Plant Breed. 126:287-290.

Liu, T., Wan, A., Liu, D., and Chen, X. 2017. Changes of races and virulence genes in Puccinia striiformis f. sp. tritici, the wheat stripe rust pathogen, in the United States from 1968 to 2009. Plant Dis. 101:1522-1532.

Mekwatanakarn, P., Kositratana, W., Levy, M., and Zeigler, R. 2000. Pathotype and avirulence gene diversity of Pyricularia grisea in Thailand as determined by rice lines near-isogenic for major resistance genes. Plant Dis. 84:60-70.

Mundt, C. 2018. Pyramiding for resistance durability: Theory and practice. Phytopathology 108:792-802. 
Pan, Q., Wang, L., Ikehashi, H., and Tanisaka, T. 1996. Identification of a new blast resistance gene in the indica rice cultivar Kasalath using Japanese differential cultivars and isozyme markers. Phythopathology 86:1071-1075.

Sun, S. 2011. Evaluation and utilization of Japanese excellent germplasm resources Tengxi (Fujikei) 138. Heilongjiang Agr. Sci. 5:4-6.

Wang, X., Jia, Y., Wamishe, Y., Jia, M., and Valent, B. 2017. Dynamic changes in the rice blast population in the USA over six decades. Mol. Plant-Microbe Interac. 30:803-812

Wu, W., Wang, L., Zhang, S., Li, Z., Zhang, Y., Lin, F., and Pan, Q. 2014. Stepwise arms race between AvrPik and Pik alleles in the rice blast pathosystem. Mol. Plant-Microbe Interac. 27:759-769.

Xin, W., Wang, J., Sun, J., Liu, H., Guo, L., Jiang, S., Xu, T., Zhao, H., and Zou, D. 2016. Physiological races of rice blast in Heilongjiang province and species identification of resistance to resource. Acta Agr. Boreali-Sinica 31:130-137.

Yang, X., Wang, L., He, Y., and Pan, Q. 2004. Molecular genetic studies on the rice blast fungus population: Dynamics of genetic and pathotypic structures of three rice blast fungus populations originated from Guangdong province in 2000 to 2002. Sci. Agric. Sinca 37:1468-1473.

Zhang, J., Sun, H., Liu, Y., Zhang, M., and Yi, T. 2009. A physiologic race on Pyricularia oryzae in Heilongjiang. Plant Prot. 35:137-140.

Zhang, S., Wang, L., Wu, W., He, L., Yang, X., and Pan, Q. 2015. Function and evolution of Magnaporthe oryzae avirulence gene $A v r P i b$ responding to the rice blast resistance gene Pib. Sci. Rep. 5:11642.

Zhang, Y. 2018. Identification of the physiological races of blast fungus in Heilongiiang province, resistance monitoring of cultivars and mapping of an avrirulence gene AvrPi12. PhD thesis. South China Agricultural University, Guangzhou, China.

Zhang, Y., Zhu, Q., Yao, Y., Zhao, Z., Correll, J., Wang, L., and Pan, Q. 2017. The race structure of the rice blast pathogen across southern and northeastern China. Rice 10:46.

Zhao, H., Wang, X., Jia, Y., Minkenberg, B., Wheatley, M., Fan, J., Jia, M. Famoso, A., Edwards, J., Wamishe, Y., Valent, B., Wang, G., and Yang, Y. 2018. The rice blast resistance gene Ptr encodes an atypical protein required for broad-spectrum disease resistance. Nat. Commun. 9:2039. 